\title{
The effect of parvalbumin deficiency on the acoustic startle response and prepulse inhibition in mice
}

\author{
Jiří Popelář ${ }^{\mathrm{a}, *}$, Natalia Rybalko ${ }^{\mathrm{a}}$, Jana Burianováa ${ }^{\mathrm{a}}$, Beat Schwaller ${ }^{\mathrm{b}}$, Josef Syka ${ }^{\mathrm{a}}$ \\ ${ }^{a}$ Institute of Experimental Medicine, Academy of Sciences of the Czech Republic, Department of Auditory Neuroscience, 14220 Prague, Czech Republic \\ ${ }^{\mathrm{b}}$ Anatomy, Department of Medicine, University of Fribourg, $\mathrm{CH}-1700$ Fribourg, Switzerland
}

\section{H I G H L I G H T S}

- PV-/- mice had smaller ASR amplitudes in response to relatively weak startling stimuli (80-90 dB SPL) in comparison with PV+/+ mice.

- PPI of the ASR in PV-/- mice was less effective than in PV+/+ mice.

- Mean ABR audiograms were found to be similar in both genotypes.

\begin{abstract}
The strength of the acoustic startle response (ASR) to short bursts of broadband noise or tone pips $(4,8$ and $16 \mathrm{kHz}$ ) and the prepulse inhibition (PPI) of the ASR elicited by prepulse tones $(4,8$ and $16 \mathrm{kHz})$ were measured in parvalbumin-deficient (PV-/-) mice and in age-matched $\mathrm{PV}+/+$ mice as controls. Hearing thresholds as determined from recordings of auditory brainstem responses were found to be similar in both genotypes. The ASRs to broadband noise and tones of low and middle frequencies were stronger than the ASRs in response to high-frequency tones in both groups. In PV-/- mice, we observed smaller ASR amplitudes in response to relatively weak startling stimuli ( $80-90 \mathrm{~dB}$ sound pressure level (SPL)) of either broadband noise or $8-\mathrm{kHz}$ tones compared to those recorded in $\mathrm{PV}+/+$ mice. For these startling stimuli, PV-/- mice had higher ASR thresholds and longer ASR latencies. PPI of the ASR in PV-/- mice was less effective than in $\mathrm{PV}+/+$ mice, for all tested prepulse frequencies $(4,8$ or $16 \mathrm{kHz})$ at $70 \mathrm{~dB}$ SPL. Our findings demonstrate no effect of PV deficiency on hearing thresholds in PV-/- mice. However, the frequency-specific differences in the ASR and the significant reduction of PPI of ASR likely reflect specific changes of neuronal circuits, mainly inhibitory, in the auditory centers in PV-deficient mice.
\end{abstract}

\section{Introduction}

Parvalbumin (PV) belongs to a large family of EF-hand calciumbinding proteins, which comprises more than 240 members in man [21]. Studies on calcium-binding protein functions indicate that these proteins are essential in $\mathrm{Ca}^{2+}$ homeostasis and for the modulation of short-lived changes in the intracellular $\mathrm{Ca}^{2+}$ concentration called $\mathrm{Ca}^{2+}$ transients. For PV, this is the case in fast-twitch muscles [23] and in a specific subpopulation of neurons [2], where PV is implicated in the subtle regulation and timing of $\mathrm{Ca}^{2+}$ signals preand postsynaptically. In the brain, PV is almost exclusively present

\footnotetext{
* Corresponding author at: Institute of Experimental Medicine AS CR, Department of Auditory Neuroscience, Videnska 1083, 14220 Prague 4, Czech Republic. Tel.: +420 241062689; fax: +420 241062787.

E-mail addresses: jpopelar@biomed.cas.cz (J. Popelář), rybalko@biomed.cas.cz (N. Rybalko), burianova@biomed.cas.cz (J. Burianová),Beat.Schwaller@unifr.ch (B. Schwaller), syka@biomed.cas.cz (J. Syka).
}

in subpopulations of inhibitory GABAergic interneurons in different brain regions including the neocortex, cerebellum, hippocampus and the reticular nucleus of the thalamus $[2-4,18]$. In the auditory system PV is expressed in cochlear hair cells [11] and in globular bushy cells' endings in the large calyx of Held on the principal cells in the medial nucleus of the trapezoid body [8]. A large number of PV-immunoreactive neurons are present in all subnuclei of the inferior colliculus, PV-positive neurons are scattered throughout layers II-VI in the auditory cortex and sparse small, oval PV-positive neurons are scattered in both the dorsal and ventral divisions of the medial geniculate body [19]. In the case of the medial geniculate body the distribution of GABA-ergic neurons is highly speciesspecific: the proportion ranges from $<1 \%$ in the bat and rat to about $25 \%$ in the cat and monkey [30]. However, only limited information exists on the role of PV in the auditory system. Several recent studies have reported age-related and region-specific changes in the expression of PV-positive neurons in a variety of mammalian species: an age-related decline of calcium binding protein-positive neurons in the dorsal cochlear nucleus of CBA/CaJ mice [13], a 
decrease in the percentage of PV-expressing neurons in the superficial layers of the auditory cortex of C57Bl/6 mice [17] or an increase of PV-positive neurons in the inferior colliculus of old Long Evans rats and a pronounced decline in the number of PV-positive neurons in the auditory cortex of aged Fischer 344 rats [19]. The assessment of the function of PV in mice has been facilitated by using genetically modified mice deficient for this protein [23]. The results obtained in in vitro studies using $\mathrm{PV}-/$ - mice suggest that PV has evolved as a functionally distinct, physiologically relevant modulator of intracellular $\mathrm{Ca}^{2+}$ transients $[22,24,25]$. Behavioral tests performed in PV-/ - mice revealed subtle alterations of locomotor function in comparison with $\mathrm{PV}+/+$ mice, characterized by a slightly increased motor activity, decreased exploratory activity and increased microlinearity of movements [7]. However, the functional consequences of PV deficiency in terms of auditory perception have not been studied until now.

In the present study, PV- - mice were used to investigate, whether the loss of PV has an effect on auditory behavior, in which both sensory and motor components play important roles. The strength of the acoustic startle reflex (ASR) and the auditory prepulse inhibition (PPI) were evaluated in $\mathrm{PV}-1-$ mice and compared with those observed in age-matched $\mathrm{PV}+/+$ mice. The ASR, defined as a transient motor response to an intense, unexpected stimulus, was used as an indicator of the behavioral responsiveness to loud sounds. The assessment of auditory PPI, i.e. the suppression of the ASR by a prepulse preceding the startling stimulus, was used as an indicator of possible changes in inhibitory function. PPI is considered to be a form of sensorimotor gating that reflects a basic inhibitory process that regulates sensory input to the brain [28]. In addition, measurements of the auditory threshold, which can influence ASR and PPI, were performed by recording auditory brainstem responses.

\section{Materials and methods}

\subsection{Animals}

Parvalbumin-deficient mice (PV-/-) were generated by homologous recombination [23] and backcrossed to C57BL/6 mice for $>10$ generations and are considered to be congenic to C57Bl/6 mice [18]. PV-/- mice ( $n=9$, mean body weight $29.0 \pm 1.6 \mathrm{~g}$ ) obtained directly from the animal facility of the University of Fribourg were genotyped by PCR [23] to validate the inactivation of the Pvalb gene. Animals were tested at five months of age and the results were compared with age-matched C57BL/6 wild type males ( $\mathrm{PV}+/+; n=10$, mean body weight $30.1 \pm 1.4 \mathrm{~g}$ ).

Mice were housed under standard conditions on a $12 \mathrm{~h} / 12 \mathrm{~h}$ light/dark cycle. The care and use of animals and all experimental procedures were performed in compliance with the guidelines of the Ethical Committee of the Institute of Experimental Medicine, Academy of Sciences of the Czech Republic, and followed the European Community Directive 86/609/EEC.

\subsection{Behavioral tests}

All behavioral tests were performed in a ventilated soundattenuated chamber (Coulbourn Habitest, model E10-21) located in a soundproof room. During the testing procedure, mice were confined to a small wire mesh cage $(85 \times 45 \times 45 \mathrm{~mm})$ on a motion-sensitive platform. The whole-body startle responses were detected and transduced by a piezoelectric accelerometer (Coulbourn E45-11B). The amplified voltage signal was acquired and processed using a RP2.1 enhanced real-time processor (Tucker Davis Technologies systemIII, Alachua, FL). The startle responses were evaluated in a 100 -ms window beginning at the onset of the startle stimulus. Acoustic startle stimuli (tone pips or noise bursts) and prepulse stimuli (tone pips) were generated by the RP2.1 enhanced real-time processor and presented via a loudspeaker (SEAS, 29AF/W) placed $12 \mathrm{~cm}$ above the platform. ASRs to 4,8 and $16 \mathrm{kHz}$ tone pips and broadband noise bursts (with a relatively flat frequency spectrum between 2 and $35 \mathrm{kHz}$ ) of 50-ms duration and varying intensity levels were recorded. In the ASR experiments, each session contained 9 trial types: a baseline trial without any stimulus and 8 startle stimuli of different intensities (50-120 dB SPL with a step size of $10 \mathrm{~dB}$ ) presented in a random order at intervals of $10-30 \mathrm{~s}$; each trial type was presented ten times. The ASR threshold, the ASR amplitude and the latency of the ASR to $110 \mathrm{~dB}$ SPL sound were analyzed.

In the PPI testing, three trial types were used: a baseline trial without any stimulus, a startling pulse alone $(115 \mathrm{~dB}$ SPL broadband noise burst of $50 \mathrm{~ms}$ ), and a combination of the startle pulse and 50-ms prepulse tones of $70 \mathrm{~dB}$ SPL, at frequencies of 4,8 and $16 \mathrm{kHz}$. The interval between the prepulse and startling stimuli was $50 \mathrm{~ms}$. All trial types were presented 10 times in pseudo-random order separated by 15-30 s. The efficacy of the PPI of ASR was expressed as: $\mathrm{PPI} \%=$ (amplitude of the ASR suppressed by the prepulse tone/amplitude of the ASR alone) $\times 100$. Thus, a PPI of $100 \%$ means no ASR suppression, a PPI of $0 \%$ corresponds to complete suppression of the ASR.

\subsection{Auditory brainstem response recordings}

The hearing threshold in mice was assessed on the basis of auditory brainstem response recordings using subcutaneous needle electrodes placed on the vertex (an active electrode) and in the neck muscles (ground and reference electrodes). Auditory brainstem responses were elicited using short-tone bursts (3-ms duration, $1 \mathrm{~ms}$ rise/fall times, frequency range $4-32 \mathrm{kHz}$ ) generated with a RP2.1 enhanced real-time processor. Acoustic stimuli were delivered in free-field conditions via a two-way loudspeaker system [Jamo ${ }^{\circledR}$ woofer (Denmark) and SEAS ${ }^{\circledR}$ T25CF 002 tweeter (Norway)] placed $70 \mathrm{~cm}$ in front of the animal's head. The acoustic system was calibrated with a Bruel\&Kjaer ${ }^{\circledR} 4939$ microphone, a ZC0020 preamplifier and a B\&K 2231 Sound Level Meter. The frequency-response curve of this system was relatively flat and varied by less than $\pm 9 \mathrm{~dB}$ between 0.15 and $40 \mathrm{kHz}$. The signal from the electrode was amplified 10,000-times, band-pass filtered over the range of $300 \mathrm{~Hz}-3 \mathrm{kHz}$, processed with a RX5-2 Pentusa Base Station (Tucker Davis Technologies systemIII, Alachua, FL) and analyzed using BioSig software. The response threshold to each frequency was determined as the minimal tone intensity that still evoked a noticeable potential peak in the expected time window of the recorded signal.

\subsection{Statistical analysis}

The differences between hearing thresholds and ASR amplitudes in PV $-/-$ mice and PV+/+ mice were tested using two-way ANOVA with the Bonferroni post-test. The differences between ASR thresholds, ASR latencies and PPI of ASR at individual frequencies were tested using an unpaired $t$-test.

\section{Results}

\subsection{Hearing thresholds}

Hearing thresholds in PV $-\mid-$ mice tended to be slightly lower (on average by $5-10 \mathrm{~dB}$ ) than the thresholds in $\mathrm{PV}+/+$ mice, but the differences were not significant ( $p>0.05$, two-way ANOVA, Bonferroni post-test). The average hearing thresholds in 5-month-old 
A

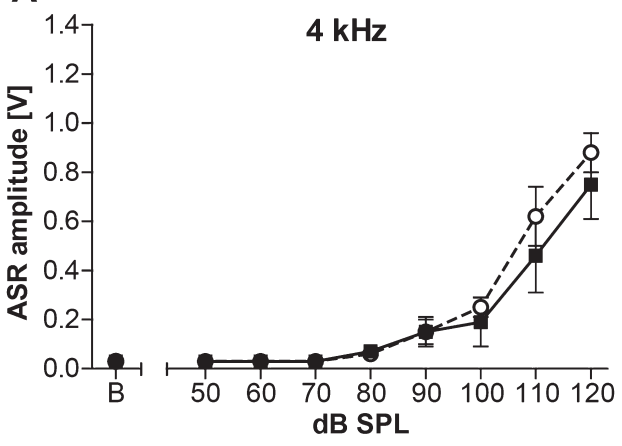

C

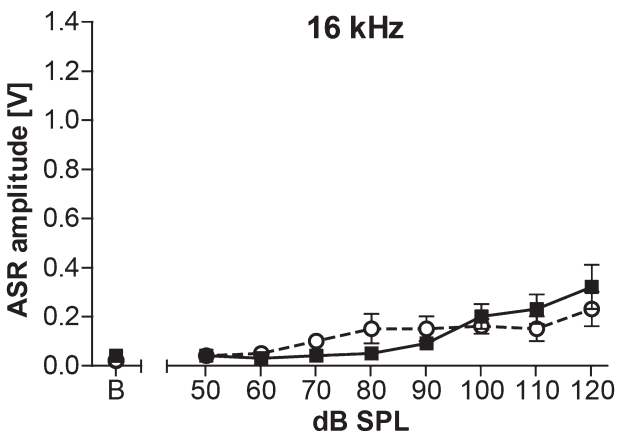

B

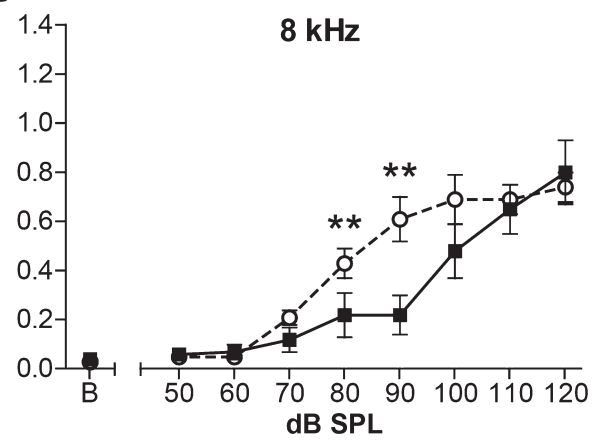

D

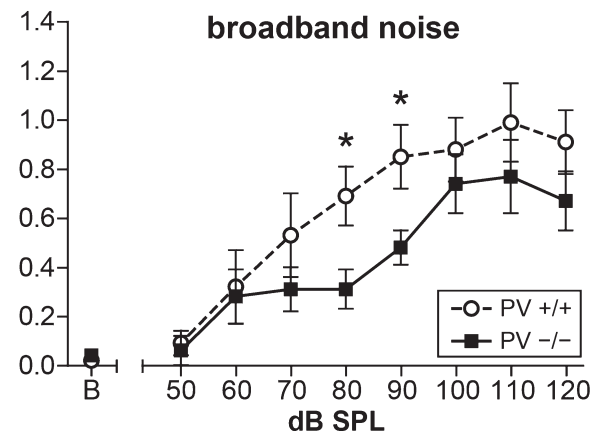

Fig. 1. Mean ASR amplitude vs. sound intensity functions in PV $-/-$ and $\mathrm{PV}+/+$ mice obtained for tone pips of 4,8 , and $16 \mathrm{kHz}$, as well as for broadband noise bursts. Bars represent \pm SEM; "B"- baseline trial; * $p<0.05,{ }^{* *} p<0.01$, two-way ANOVA with the Bonferroni post-test.

$\mathrm{PV}-/-$ and $\mathrm{PV}+/+$ mice are shown in Supplementary material in Fig. S1.

\subsection{Acoustic startle reflex (ASR)}

The dependence of the ASR amplitude on the startling stimulus intensity for PV $-/-$ and PV+/+ mice is shown in Fig. 1.

The ASRs to broadband noise and tones of 4 and $8 \mathrm{kHz}$ (Fig. 1A, $\mathrm{B}$ and $\mathrm{D})$ were generally stronger than the ASRs to $16-\mathrm{kHz}$ tones (Fig. 1C) in all mice. The differences between the ASRs in PV-/and $\mathrm{PV}+/+$ mice were clearly evident in response to broadband noise and to tones of $8 \mathrm{kHz}$ : the ASR amplitudes evoked by these startling stimuli were smaller in $\mathrm{PV}-/$ - than in $\mathrm{PV}+/+$ mice (Fig. $1 \mathrm{~B}$ and $\mathrm{D}$ ), but significant differences were only observed at 80 and $90 \mathrm{~dB} \mathrm{SPL}(p<0.05$ and $p<0.01$, respectively, two-way ANOVA with the Bonferroni post-test). In addition, PV- - - mice had higher ASR thresholds than did PV+/+ animals, but differences were significant only at $8 \mathrm{kHz}(78.9 \pm 6.0 \mathrm{~dB}$ SPL vs. $68.0 \pm 4.7 \mathrm{~dB}$ SPL, resp., $p<0.01$, unpaired $t$-test $)$ and $16 \mathrm{kHz}(87.5 \pm 4.2 \mathrm{~dB}$ SPL vs. $78.5 \pm 6.6 \mathrm{~dB}$ SPL, resp.; $p<0.05$, unpaired $t$-test).

The latencies of the first positive wave of the ASR evoked by 110-dB startle stimuli were analyzed (Table 1 ). PV-/ - mice exhibited longer ASR latencies in comparison to $\mathrm{PV}+/+$ mice. Despite the fact that their amplitudes, evoked by $110 \mathrm{~dB}$ SPL startle stimuli, were similar, the differences in latencies were significant at 4 and

Table 1

Average latencies of the ASR in PV $-/-$ and $\mathrm{PV}+/+$ mice (mean $\pm \mathrm{SEM}) .{ }^{*} p<0.05$ ${ }^{* *} p<0.01$, unpaired $t$-test.

\begin{tabular}{llll}
\hline Startle stimulus (110 dB SPL) & PV $-/-$ & & PV+/+ \\
\hline Broadband noise & $25.0 \pm 0.6 \mathrm{~ms}$ & $\mathrm{~ns}$ & $25.1 \pm 0.6 \mathrm{~ms}$ \\
$4 \mathrm{kHz}$ & $26.2 \pm 0.5 \mathrm{~ms}$ & ${ }^{* *}$ & $25.3 \pm 0.4 \mathrm{~ms}$ \\
$8 \mathrm{kHz}$ & $25.9 \pm 0.9 \mathrm{~ms}$ & $*$ & $24.7 \pm 0.7 \mathrm{~ms}$ \\
$16 \mathrm{kHz}$ & $26.4 \pm 0.7 \mathrm{~ms}$ & $\mathrm{~ns}$ & $25.5 \pm 1.4 \mathrm{~ms}$ \\
\hline
\end{tabular}

$8 \mathrm{kHz}\left({ }^{*} p<0.05\right.$ and ${ }^{* *} p<0.01$, resp., unpaired $t$-test). The ASR latencies evoked by broadband noise were similar in both experimental groups of mice.

\subsection{Prepulse inhibition (PPI) of ASR}

The effect of modifying the ASR amplitudes by a preceding stimulus was tested using PPI of ASR in mice of both genotypes. Fig. 2 shows the changes in the average relative ASR amplitudes evoked by $115 \mathrm{~dB}$ SPL broadband noise stimuli, using prepulse stimuli at 4 , 8 and $16 \mathrm{kHz}$ at an intensity of $70 \mathrm{~dB}$ SPL in PV-/- and PV+/+ mice; a lower relative ASR amplitude corresponds to stronger PPI (from no inhibition at $100 \%$ to complete ASR inhibition at $0 \%$ ). The results document a significantly less effective PPI in PV $-/-$ mice than in $\mathrm{PV}+/+$ controls at all tested PPI frequencies $\left({ }^{*} p<0.05,{ }^{* * *} p<0.001\right.$, unpaired $t$-test).

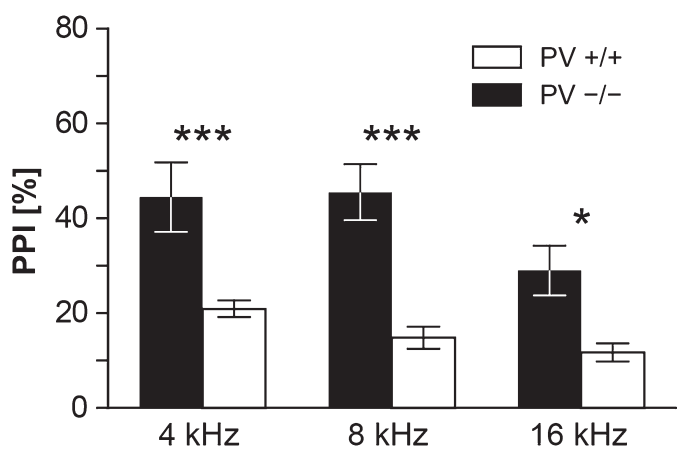

Fig. 2. The efficacy of PPI of ASR in PV $-/-$ and PV+/+ mice. The efficacy of the PPI of ASR was expressed as: PPI = (ASR amplitudes evoked by $115 \mathrm{~dB}$ SPL broadband noise stimuli and suppressed by the prepulse tone at 4,8 or $16 \mathrm{kHz}$ at an intensity of $70 \mathrm{~dB}$ SPL/amplitude of the ASR alone) $\times 100 \%$. A PPI of $0 \%$ corresponds to complete ASR suppression. ${ }^{*} p<0.05,{ }^{* * *} p<0.001$, unpaired $t$-test), bars represent \pm SEM. 


\section{Discussion}

The results of our study demonstrate that the absence of PV in PV-/- mice altered ASR parameters and that these mice showed significantly less efficient prepulse inhibition of the ASR in comparison to $\mathrm{PV}+/+$ mice with normal $\mathrm{PV}$ levels. The average hearing thresholds in PV $-/-$ mice were, however, comparable with those found in $\mathrm{PV}+/+$ mice. The calcium-binding protein $\mathrm{PV}$ is present in both the inner and outer hair cells in the cochlea, and its role is to modulate $\mathrm{Ca}^{2+}$ entry to the hair cell cytoplasm during mechanoelectrical transduction and afferent and efferent synaptic activity [10]. Due to the similarity of the hearing thresholds in PV-/- and $\mathrm{PV}+/+$ mice, the role of $\mathrm{PV}$ in $\mathrm{Ca}^{2+}$ buffering does not seem to be essential for the subject's hearing sensitivity near the threshold.

The ASR, as an unconditioned reflex reaction to an abrupt loud sound, is represented by a short neural circuit comprising the posteroventral cochlear nucleus, one or more nuclei of the lateral lemniscus, the caudal pontine reticular nucleus and spinal interneurons and motor neurons $[5,15]$. In spite of its relative simplicity, the ASR shows several forms of behavioral plasticity (habituation, facilitation, prepulse inhibition and modification by prior associative learning) that reflect the processing of acoustic information at higher levels of the auditory system than the neural circuit of the ASR itself $[14,15,20]$. These features of the startle reflex allow the study of not only the processing of auditory stimuli, but also of complex brain function, including movement control or cognitive disorders.

Our results indicate that the absence of PV induces "abnormalities" in the neural processes that finally result in changes of the startle reflex formation. The significantly longer ASR latency, found in our study in PV-deficient mice compared with $\mathrm{PV}+/+$ mice, might be related to the prolongation of the time required to attain peak force and the increased force produced during a single muscle twitch [23], or it could be linked to a mild impairment in locomotor coordination that has been observed in PV-/- mice [7]. The most distinct differences in ASR parameters, i.e. significantly higher ASR thresholds, lower ASR amplitudes and longer ASR latencies in $\mathrm{PV}-/-$ mice in comparison with $\mathrm{PV}+/+$ mice, were observed using $8-\mathrm{kHz}$ tones. This is not surprising given that the hearing sensitivity was highest and the reflex response was more pronounced (especially in comparison with $16 \mathrm{kHz}$ ) in both genotypes at this frequency (Fig. S1, Fig. 1). These results suggest that ASR differences reflect not only parvalbumin deficiency in the skeletal muscles, but most likely also specific changes in the auditory neuronal circuits.

The PPI of ASR represents a basic inhibitory process regulating sensory inputs, and the magnitude of PPI can serve as a measure for the behavioral salience of perceptually-relevant stimuli $[9,29]$. Previous animal studies have shown that for PPI of ASR, several pathways are implicated including direct glutamatergic projections from the cochlear nuclei, inhibitory cholinergic projections from the pedunculopontine tegmental nucleus and indirect inputs from the superior and inferior colliculi [15]. Electrophysiological recordings of cortical, hippocampal and cerebellar neuronal activity in PV-/ - mice in vitro showed that the absence of PV in a subpopulation of GABA-ergic interneurons resulted in modification of the dynamics of inhibitory control (for reviews, see [22]), enhancement of the severity of pharmacologically-induced seizures [25], alterations of Purkinje cell-firing [26], and modulation of the firing properties of the reticular thalamic nucleus bursting neurons [1]. Fast-spiking PV-positive inhibitory cells, which represent the largest inhibitory subpopulation in the auditory cortex of the cat, are involved in the sharpening of frequency and intensity tuning and the shaping of spectral modulation preferences [27,31]. However, little is known so far about the influence of the missing parvalbumin on neuronal activity in the central auditory nuclei in PV-/- mice. The results of animal studies have suggested that for the PPI of ASR, the cochlear nucleus, the superior and inferior colliculi and the pedunculopontine tegmental nucleus are important $[15,29]$, and PPI can be modulated by the auditory cortex, amygdala, hippocampus and striatum [29]. Our data clearly demonstrate that the effect of PPI on ASR is much weaker in PV-/- mice than in controls. This could be linked to the decreased density of PVpositive inhibitory interneurons, possibly as the result of reduced PV expression levels, in the auditory nuclei of some mouse and rat strains during aging $[13,19]$. In line, in dystonic hamsters showing a transient decrease in PV-positive neurons and/or PV levels at approximately 30 days of age, ASR is decreased [11]. The low efficiency of PPI in PV-/- mice could be also related to altered function of the cortico-tectal pathways, which are under the strong influence of parvalbumin interneurons in the auditory cortex [6].

At the behavioral level, the testing of PPI of ASR has been used for detecting inhibitory alterations in various sensori-motor systems. A deficiency or even the absence of PPI of ASR was observed in mice with $G_{A B A}$ receptor $\gamma 2$ subunits removed from the PVpositive neurons [16], in GAD65 knockout mice [12] and in dystonic hamsters [11].

In the present study, a reduced PPI was observed in PV-/- mice at all prepulse stimulus frequencies, i.e. 4,8 and $16 \mathrm{kHz}$. Because the hearing thresholds were similar in both genotypes, the observed differences with respect to auditory behavior (ASR and PPI of ASR) are not directly related to peripheral hearing sensitivity. Our data support the idea that the absence of PV in the central auditory nuclei is responsible for changes in the inhibitory system resulting in the reduction of PPI of ASR. For investigating specific changes in the central auditory system of PV-/- mice, electrophysiological experiments and more sophisticated behavioral tests using conditioning procedures should be performed.

In conclusion, the results of our study demonstrate no effect of PV deficiency on the auditory periphery, resulting in unaltered hearing thresholds. The frequency-specific differences in the ASR and the significant reduction of PPI of ASR in PV-deficient mice likely reflect specific changes of neuronal circuits and/or circuit function, mainly inhibitory, in the auditory centers in PV-deficient mice.

\section{Conflict of interest}

The authors have declared that no conflict of interest exists.

\section{Acknowledgments}

This work was supported by the Grant P304/12/1342 from the Grant Agency of the Czech Republic and by the project OP RDI CZ.1.05/1.1.00/02.0109 "Biotechnology and Biomedicine Center of the Academy of Sciences and Charles University in Vestec (BIOCEV)", Operational Program Research and Development for Innovations by the Ministry of Education, Youth and Sports of the Czech Republic and the European Regional Development Fund.

\section{Appendix A. Supplementary data}

Supplementary data associated with this article can be found, in the online version

\section{References}

[1] L. Alberi, A. Lintas, R. Kretz, B. Schwaller, A.E. Villa, The calcium-binding protein parvalbumin modulates the firing properties of the reticular thalamic nucleus bursting neurons, J. Neurophysiol. 109 (2013) 2827-2841.

[2] M.R. Celio, Calbindin D-28k and parvalbumin in the rat nervous system, Neuroscience 35 (1990) 375-475. 
[3] M.R. Celio, C.W. Heizmann, Calcium-binding protein parvalbumin as a neuronal marker, Nature 293 (1981) 300-302.

[4] R. Coveñas, M. De León, J.R. Alonso, R. Arévalo, J. Lara, J. Aijón, Distribution of parvalbumin-immunoreactivity in the rat thalamus using a monoclonal antibody, Arch. Ital. Biol. 129 (1991) 199-210.

[5] M. Davis, D.S. Gendelman, M.D. Tischler, P.M. Gendelman, A primary acoustic startle circuit: lesion and stimulation studies, J. Neurosci. 2 (1982) 791-805.

[6] R. Druga, J. Syka, Ascending and descending projections to the inferior colliculus in the rat, Physiol. Bohemoslov. 33 (1984) 31-42.

[7] M.A. Farre-Castany, B. Schwaller, P. Gregory, J. Barski, C. Mariethoz, J.L. Eriksson, I.V. Tetko, D. Wolfer, M.R. Celio, I. Schmutz, U. Albrecht, A.E. Villa, Differences in locomotor behavior revealed in mice deficient for the calcium-binding proteins parvalbumin, calbindin D-28k or both, Behav. Brain Res. 178 (2007) 250-261.

[8] F. Felmy, R. Schneggenburger, Developmental expression of the $\mathrm{Ca}^{2+}$-binding proteins calretinin and parvalbumin at the calyx of Held of rats and mice, Eur. J. Neurosci. 20 (2004) 1473-1482.

[9] M.A. Geyer, D.L. Braff, Startle habituation and sensorimotor gating in schizophrenia and related animal models, Schizophr. Bull. 13 (1987) 643-668.

[10] C.M. Hackney, S. Mahendrasingam, A. Penn, R. Fettiplace, The concentrations of calcium buffering proteins in mammalian cochlear hair cells, J. Neurosci. 25 (2005) 7867-7875,

[11] M. Hamann, M. Bennay, M. Gernert, K. Schwabe, M. Koch, A. Richter, Decreased density of amygdaloid parvalbumin-positive interneurons and behavioral changes in dystonic hamsters (Mesocricetus auratus), Behav. Neurosci. 122 (2008) 36-43.

[12] S.A. Heldt, A. Green, K.J. Ressler, Prepulse inhibition deficits in GAD65 knockout mice and the effect of antipsychotic treatment, Neuropsychopharmacology 29 (2004) 1610-1619

[13] E. Idrizbegovic, B. Canlon, L.S. Bross, J.F. Willott, N. Bogdanovic, The total number of neurons and calcium binding protein positive neurons during aging in the cochlear nucleus of CBA/CaJ mice: a quantitative study, Hear. Res. 158 (2001) $102-115$.

[14] J.R. Ison, G.R. Hammond, Modification of the startle reflex in the rat by changes in the auditory and visual environments, J. Comp. Physiol. Psychol. 75 (1971) 435-452.

[15] M. Koch, The neurobiology of startle, Prog. Neurobiol. 59 (1999) 107-128.

[16] E. Leppä, A.M. Linden, O.Y. Vekovischeva, J.D. Swinny, V. Rantanen, E. Toppila, H. Höger, W. Sieghart, P. Wulff, W. Wisden, E.R. Korpi, Removal of GABA(A) receptor $\gamma 2$ subunits from parvalbumin neurons causes wide-ranging behavioral alterations, PLoS one 6 (2011) e24159.

[17] H.N. Martin del Campo, K.R. Measor, K.A. Razak, Parvalbumin immunoreactivity in the auditory cortex of a mouse model of presbycusis, Hear. Res. 294 (2012) 31-39.
[18] H. Moreno, N.S. Burghardt, D. Vela-Duarte, J. Masciotti, F. Hua, A.A. Fenton, B. Schwaller, S.A. Small, The absence of the calcium-buffering protein calbindin is associated with faster age-related decline in hippocampal metabolism, Hippocampus 22 (2012) 1107-1120.

[19] L. Ouda, R. Druga, J. Syka, Changes in parvalbumin immunoreactivity with aging in the central auditory system of the rat, Exp. Gerontol. 43 (2008) 782-789.

[20] N. Rybalko, Z. Bureš, J. Burianová, J. Popelář, J. Grécová, J. Syka, Noise exposure during early development influences the acoustic startle reflex in adult rats, Physiol. Behav. 102 (2011) 453-458.

[21] B. Schwaller, The continuing disappearance of "pure" $\mathrm{Ca}^{2+}$ buffers, Cell. Mol. Life Sci. 66 (2009) 275-300.

[22] B. Schwaller, The use of transgenic mouse models to reveal the functions of $\mathrm{Ca}^{2+}$ buffer proteins in excitable cells, Biochim. Biophys. Acta 1820 (2012) 1294-1303.

[23] B. Schwaller, J. Dick, G. Dhoot, S. Carroll, G. Vrbova, P. Nicotera, D. Pette, A. Wyss, H. Bluethmann, W. Hunziker, M.R. Celio, Prolonged contraction-relaxation cycle of fast-twitch muscles in parvalbumin knockout mice, Am. J. Physiol. 276 (1999) C395-C403.

[24] B. Schwaller, M. Meyer, S. Schiffmann, 'New' functions for 'old' proteins: the role of the calcium-binding proteins calbindin D-28k, calretinin and parvalbumin, in cerebellar physiology. Studies with knockout mice, Cerebellum 1 (2002) 241-258.

[25] B. Schwaller, I.V. Tetko, P. Tandon, D.C. Silveira, M. Vreugdenhil, T. Henzi, M.C. Potier, M.R. Celio, A.E. Villa, Parvalbumin deficiency affects network properties resulting in increased susceptibility to epileptic seizures, Mol. Cell. Neurosci. 25 (2004) 650-663.

[26] L. Servais, B. Bearzatto, B. Schwaller, M. Dumont, C. De Saedeleer, B. Dan, J.J. Barski, S.N. Schiffmann, G. Cheron, Mono- and dual-frequency fast cerebellar oscillation in mice lacking parvalbumin and/or calbindin D-28k, Eur. J. Neurosci. 22 (2005) 861-870

[27] M.L. Sutter, C.E. Schreiner, M. McLean, K.N. O‘connor, W.C. Loftus, Organization of inhibitory frequency receptive fields in cat primary auditory cortex, J. Neurophysiol. 82 (1999) 2358-2371.

[28] N.R. Swerdlow, D.L. Braff, M.A. Geyer, Animal models of deficient sensorimotor gating: what we know, what we think we know, and what we hope to know soon, Behav. Pharmacol. 11 (2000) 185-204.

[29] N.R. Swerdlow, M.A. Geyer, D.L. Braff, Neural circuit regulation of prepulse inhibition of startle in the rat: current knowledge and future challenges, Psychopharmacology (Berl) 156 (2001) 194-215.

[30] J.A. Winer, D.T. Larue, Evolution of GABAergic circuitry in the mammalian medial geniculate body, Proc. Natl. Acad. Sci. U.S.A. 93 (1996) 3083-3087.

[31] K. Yuan, J.Y. Shih, J.A. Winer, C.E. Schreiner, Functional networks of parvalbumin-immunoreactive neurons in cat auditory cortex, J. Neurosci. 31 (2011) 13333-13342. 